\title{
Guided Filter Smoothing for Third Order Edge Mask
}

\author{
G.J.Joyce Mary, PhD \\ Associate Professor,Computer Science Engg. \\ PRIST University \\ Thanjavur, S.India
}

\author{
A.Rijuvana Begum \\ Research Scholar \\ PRIST University \\ Thanjavur, S.India
}

\begin{abstract}
Images corrupted by the noise during either transmission or acquisition process. Filtering and or smoothing an important task to reduce the effect of noises that contaminates images. Hence the processed image is useful for further applications. This paper analyses the suitable smoothing filter for the edge mask derived using Third Order Difference Equation (TODE). State of the art edge preserving smoothing filters like non linear bilateral filter and linear guided filter is applied on different sample images after degrading them with Additive White Gaussian Noise(AWGN). Since AWGN is most common type of Gaussian noise useful for testing. Both second and third order edge mask is tested using these approaches. Performance metric such as MSE, PSNR and Entropy were computed. The results prove that the guided filter is best suited for the TODE edge mask.
\end{abstract}

\section{Keywords}

Edge detection, Third Order Difference Equation (TODE), Edge Mask, Smoothing, Bilateral filter, Guided Filter.

\section{INTRODUCTION}

Many Machine vision operations require smoothing as a pre processing step to reduce image noise. To blur the image and reduce the noise smoothing filters are used. Small detail in an image is removed by blurring and the gaps are bridged with lines and curves. Thus the noise removal can be achieved by blurring and linear or non linear filtering [1]. Images are often corrupted by various types of noise such as Impulse noise, Random noise, Poisson noise and Gaussian noise. Impulse noise is of two forms, namely random valued impulse noise and salt and pepper noise. To calculate the smoothed value at a pixel, a square grid of neighborhood centered at the pixel is averaged [2]. Filters provide an aid to visual analysis of images for further digital image processing applications such as segmentation, compression, registration etc. Noise removal can be achieved by using a number of existing linear filtering techniques which are mathematically simple. Filters are linear if the output values are linear combinations of the pixels in the original image, otherwise they are nonlinear. Linear Filters are well defined and computationally fast, but are not capable to smooth without simultaneously blurring edges. Nonlinear filters can smooth without blurring edges and can detect edges at all directions concurrently, but have not as much of secure theoretical foundations and is slow to compute [3][4].

\section{RELATED WORK}

Image denoising and edge detection is performed on cold trap radiographic images. Mean, Median filters used for denoising and Roberts, Sobel, Prewitt, Laplacian, LOG and Canny were used for edge detection. Results show that Median Filter, LOG and Canny work better on radiographic images [5].

Gaussian, Speckle, Salt and Pepper and Poisson noise added to the original lena image .Wiener, Mean and Median filters were used for noise removal. Simulation performance shows that Weiner filter works well for Poisson and speckle noise than mean and median filters [6].

Non Local Mean (NLM) algorithm based on the concept of self similarity is used to denoise the images corrupted by the noise. Results proves that NLM algorithm perform better than Gaussian and Weiner filter [7].

Speckle noise reduction for ultra sound medical images are performed by all standard speckle filters which causes over smoothing artifacts. This effect is overcome by contourlet transform which is directly defined on discrete rectangular grid is best suited for speckle reduction in ultra sound images[8].

Colour image enhancement and smoothing is done by using non linear filters such as Edge Preserving Smoothing (EPS), Symmetric Nearest Neighbour (SNN) and Vector Median Filter (VMF).Parameters like SNR and Colour difference metric used for analysis. Resultant performance proves that VMF outperforms than EPS and SNN [9].

Electrocardiographic images corrupted by speckle noise are removed using common spatial filters like Mean, Median, Gaussian and Laplacian. Three statistical measures like RMSE, SNR and PSNR were evaluated. The experimental results prove that the Laplacian is best suited for noise reduction and detail enhancement for further processing applications [10].

Generalized Value and Criterion Filter Structure (VCFS) with different weighting function used for preserving both corners and edges while smoothing. This operation has been performed over wide variety of both Gray scale and colour images to achieve painting like artistic effects [11].

Bilateral Filter is a non linear filtering technique to smooth images while preserving edges. It is based on the principle that each pixel is replaced by its weighted average of its neighbours and it depends on two parameters that indicate the size and contrast of the feature to preserve. Performance of the result is compared with the iterative approaches such as Anisotropic Diffusion (AD), Weighted Least Squares (WLS) and Robust Estimation (RE) conclude that the bilateral filter is a best non iterative edge preserving non linear filter [12].

Guided Image filter used for preserving edges which is an explicit filter derived based on local linear model in which the output filtered image is based on the guidance image which may be either input image itself or any other image. It is the recent fastest edge preserving filter which removes the gradient reversal artifacts occurred in bilateral filter [13]

Local Linear Stein's Unbiased Risk Estimate (LLSURE) to preserve high quality edges and fine details in an image. It is a simple, less computationally complex and the design parameters are automatically tuned for the requisite. The 
experimental results show that this approach is robust and flexible for wide variety of images [14].

A new edge preserving smoothing technique that combines $\mathrm{L}_{0}$ gradient minimization technique with average filtering, Alpha blending, Unsharp masking and Contrast adjustment used in color images. Metrics PSNR, Structural Similarity Index and Gradient are computed for different color images. Performance result shows that the proposed method outperforms than the existing $\mathrm{L}_{0}$ gradient minimization technique [15].

\section{NOISE TYPES}

Noise in image is a consequence from the random fluctuations in intensity or color information. Noise produces the undesirable information which affects the quality of the image. Noise is defined as a process which affects the acquired image quality that is being not a part of the original image content. Digital image noise may occur due to various sources. During acquisition process, during digital images conversion, and image transmission. In practice image noise is regarded as an undesirable derivative of image capture. Following are the some common types of noises [16].

- Gaussian noise or Amplifier noise

- Poisson noise or Shot noise

- Salt-and-pepper noise

- Speckle noise

\subsection{Gaussian noise}

The main source for this noise is that it occurs during image acquisition process that is in image sensor due to poor illumination (sensor noise) and during image transmission. It is a standard and commonly used noise type for analysis. It is otherwise called as normal noise and is evenly distributed over the image means that each pixel in the noisy image is the sum of true pixel value and random Gaussian noise. One common form of Gaussian noise is Additive White Gaussian Noise (AWGN) [16] [17].

\subsection{Shot noise}

Shot noise caused by statistical quantum variations, that is, disparity in the number of photons sensed at a given exposure level. This noise is known as photon shot noise. Shot noise has a root-mean-square value proportional to the square root of the pixel intensity, and the noises at different pixels are independent [16].

\subsection{Salt and Pepper noise}

It is an impulse type noise generally caused by malfunctioning of pixel elements in the camera sensors, defective memory locations, or timing errors in the digitization process. The noisy image has black pixels on bright regions and white pixels on dark regions thus giving the appearance like salt and pepper [17] [18].

\subsection{Speckle noise}

Speckle noise is multiplicative type of granular noise occurs due to unsystematic interference between coherent return signals from an object. This type of noise degrades the imageries sensed from coherent imaging systems like SAR (Synthetic Aperture Radar), laser and ultrasound [17].Speckle noise is a more serious issue, causing difficulties for the observer to differentiate fine details of the images in diagnostic purposes. It has gamma distribution. Thus, denoising has become a predominant step in medical image processing [19].

\section{CLASSICAL DENOISING TECHNIQUES}

Image denoising is the key concepts in imaging and computing field. Image denoising manipulates the noisy image to fabricate a visually appealing high quality image. It is widely used in various image-related applications such as three dimensional object detection, MRI analysis, CT images etc. The images degraded by some degree of noise due to image sensing phenomenon and transmission process. The aim of image denoising is to recover the actual image by removing undesirable noise with the constraint that low computational complexity and edge preserving ability [7] Many denoising techniques in existence. Some of the common denoising techniques are mean filtering, Weiner filtering, Gaussian filtering, median filtering and latest techniques like bilateral filtering, guided image filtering. This section discusses the behavior of above mentioned techniques in detail.

\subsection{Mean filter}

Mean filter is the simplest form of smoothing filters. It is also called as averaging filter. Mean filter reduces the amount of intensity variation between one pixel and the next. Basic idea is that it uses a mask which operates over each pixel in an image to calculate its average value surrounded by the mask. This average value replaces the actual pixel.

$$
\text { Mean Filter of }\left(Z_{1}, Z_{2}, Z_{3} \ldots \ldots . Z_{N}\right)=\frac{1}{N} \sum_{\mathrm{i}=1}^{N} Z_{\mathrm{i}}
$$

Where $\left(Z_{1}, Z_{2} \ldots . . Z_{N}\right)$ is range of image pixel. Mean filter is useful for eliminating grain noise from the photography image. But it fails to preserve edges in the image. There are two main problems with the mean filters, which are [19] [20].

i. Single pixel with misleading affects the average value of all the neighborhood pixels.

ii. If the neighborhood of the filter overlaps an edge, the filter will blur it.

\subsection{Weiner filter}

Weiner filter is a linear filter works based on statistical approach. A Wiener filter is a least mean square error filter. This filter requires knowledge about image and noise spectra for its operation. It recovers the original image in the presence of both noise and blur. It computes image variance to perform smoothing. Higher the variance lesser will be the smoothing and vice versa. Hence for better smoothing the variance should be small. It performs better than mean filter interms of preserving edges and other high frequency detail information. Main drawback of this method is that it takes longer computational time than mean filter [8] [20].

In general Wiener filters follows some properties like noise and original image is assumed to be stationary linear random processes with known power spectral density, the filter must be causal and the filter operates with the constraint that minimum mean-square error [22].

Operation of Wiener filter is defined as,

Where,

$$
Z(u, v)=H^{*}(u, v) /\left\{|H(u, v)|^{2}+P_{N}(u, v) / P_{I}(u, v)\right\}
$$

$\mathrm{H}(\mathrm{u}, \mathrm{v}) \quad=$ Filter transfer function in transform

domain

$\mathrm{H}^{*}(\mathrm{u}, \mathrm{v}) \quad=$ Complex conjugate of $\mathrm{H}(\mathrm{u}, \mathrm{v})$

$\mathrm{P}_{\mathrm{N}}(\mathrm{u}, \mathrm{v}) \quad=$ Noise Power Spectral Density

$\mathrm{P}_{\mathrm{I}}(\mathrm{u}, \mathrm{v}) \quad$ = Image Power Spectral Density 


\subsection{Median filter}

It is a typical non linear filter that replaces every pixel in an image by the median of the gray values of pixels in a specific neighborhood surrounded by the mask. If the mask contains odd number of entries then the median is the middle value of gray level otherwise for even number of entries average between two central gray values considered as the median .The median filter is also called the order statistics filter because it is based on arranging the pixels in ascending order to calculate its median. It is a popular filter used to reduce salt and pepper noise and speckle noise without blurring edges of the image [21]. One of the major problem with the median filter is that it is relatively expensive and complex computation. Also median filter removes both noise and fine details in the image since it cannot distinguish them [8].

\subsection{Gaussian filter}

Gaussian filter is a separable and circularly symmetric filter. Filtering based on peak detection. The peaks are the impulses in the image. This filter adjusts and corrects both spectral coefficient and amplitude spectrum surrounded by the filter window. Important properties of Gaussian filter include [6] [16],

1. They are linear Low Pass Filters (LPF) reduces edge blurring.

\section{Higher computational efficiency.}

3 The degree of smoothing is controlled by $\sigma$ ( $\sigma$ defines the size of the neighborhood and larger $\sigma$ for more intensive smoothing).

\subsection{Bilateral filter}

It is a simplest explicit weighted average non linear filter for preserving edges while smoothing. The filtered output at each pixel is the Gaussian weighted average of neighboring pixels of both spatial and gray level range. Joint bilateral filter is the generalization of bilateral filter in which the weights are obtained from another guidance image. Though the bilateral filter is a state of the art edge preserving smoothing filter its main drawback is 'gradient reversal 'artifacts. It is mainly due to constant Gaussian weighted average in the image areas of similar gray level values. The two parameters controlling the behavior of the filter are range kernel standard deviation and domain kernel standard deviation. Bilateral filter is a non iterative filter and cost effective approach. Bilateral filter decompose the input image in to two components namely filtered output (minute edge textures) and residual image (noise texture) [23][24]

\subsection{Guided filter}

It is a linear rotation variant explicit filter in which the filtered output is obtained by considering the guidance image which may be either input image itself or any other image. The filtered output is independent of kernel size and intensity range. It is the most reliable filter for noisy images. It also preserves edges while smoothing .Its other applications includes image matting, detail enhancement, image denoising and HDR compression. The operation of guided image filtering is shown in fig.1.Window radius ' $r$ ' and regularization parameter $\xi$ are the two important design functions to implement filtering operation. Guided filter overcomes the gradient reversal problem of bilateral filter.

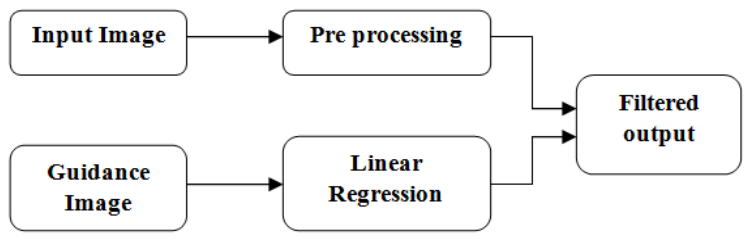

Fig.1. Guided Image filter

Window radius ' $r$ ' takes different values as 2,4and 8 .Similarly the regularization parameter ' $\xi$ ' considered as $0.1^{2}, 0.2^{2}$ and $0.4^{2}[23]$.

\section{TRADITIONAL EDGE DETECTION METHODS}

Edge detection is a fundamental step in mage analysis and segmentation. Edges are defined as the set of pixels with sharp changes in its gray level values or color. Edges form the boundary between the objects. Edge detection incorporates five steps, which are Smoothing, Filtering, Enhancement, Detection and Localization. The process of edge detection reduces the amount of data to be processed further for wide range of applications such as Image compression, Target tracking, Object recognition etc.[25]. This section describes about three basic edge operators to determine the edge map.

\subsection{First Order Edge Operators}

First order edge detection masks are the simple and oldest operators to detect the change in image intensity or color of an image. Robert, Sobel and Prewitt are the commonly used masks to determine the image gradients in vertical and horizontal directions. The edge map is obtained by convolving image with masks shown in fig. 2 through fig. 4 .

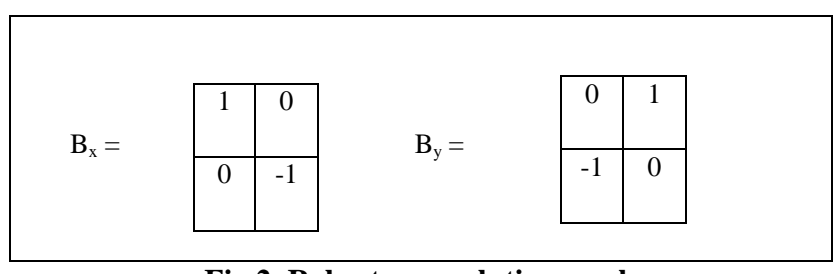

Fig.2. Roberts convolution mask

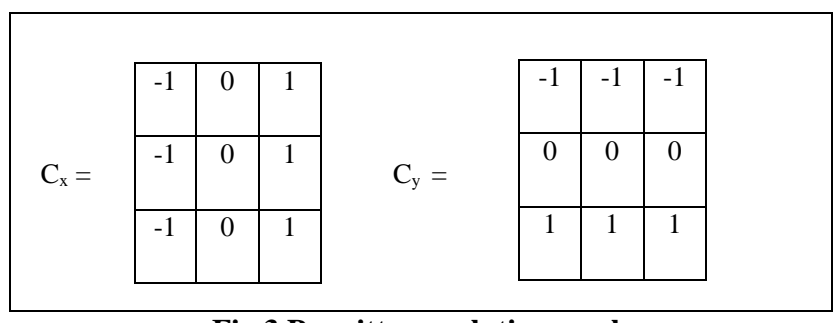

Fig.3.Prewitt convolution mask

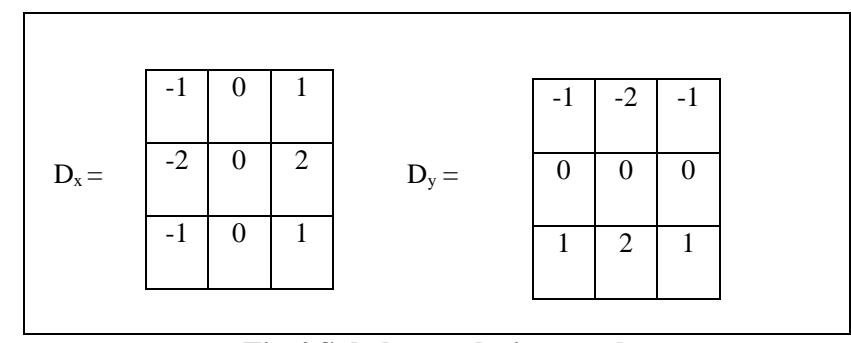

Fig.4.Sobel convolution mask 
Advantages:

$\checkmark \quad$ Simple to implement.

$\checkmark$ Orientations and edges are easily detected.

Drawbacks:

$\checkmark \quad$ Produces thick edges.

$\checkmark$ Poor localization and inaccurate.

\subsection{Second Order Edge operator}

Laplacian is the second order edge mask. It is a single isotropic (rotation independent) mask to detect edges. Its zero crossing property detects the location of an edge. Laplacian convolution mask is of size $3 \times 3$ shown in fig. 5 .

\begin{tabular}{|c|c|c|}
\hline 0 & -1 & 0 \\
\hline-1 & 4 & -1 \\
\hline 0 & -1 & 0 \\
\hline
\end{tabular}

Fig.5.Laplacian convolution mask

Advantages:

$\checkmark \quad$ Less computation time, since it involves singe mask compared to first order operator.

$\checkmark \quad$ It produces thinner edges.

Disadvantage

$\checkmark \quad$ It is highly sensitive to noise because it is a second derivative.

\subsection{Third Order Edge Operators}

To improve the performance of the second order Laplacian, a new operator of size $4 \times 4$ is derived using Third Order Difference Equation (TODE).This mask provides higher PSNR and lower MSE values for different images and file formats. The mask is shown in fig.6.

\begin{tabular}{|c|c|c|c|}
\hline 0 & 0 & -1 & 0 \\
\hline 0 & 0 & 3 & 0 \\
\hline-1 & 3 & -6 & 1 \\
\hline 0 & 0 & 1 & 0 \\
\hline
\end{tabular}

Fig.6.Third order convolution mask

Advantage

$\checkmark \quad$ Offers high Peak Signal to Noise Ratio(PSNR) and low Mean Square Error(MSE)

Disadvantage

$\checkmark \quad$ Superior noise sensitivity. Since the edge pixel is differentiated thrice [25] [26].

\section{PROPOSED WORK}

The edge mask simply enhances and sharpens the edge pixels. Since both noise and edges are high frequency components the masks enhances both edge and noise pixels [25]. Hence appropriate smoothing technique is used to remove noise before applying the edge mask. Various edge preserving smoothing techniques are available in domain, out of which the state of the art techniques are non linear bilateral filter and linear guided filter. In this section different images and file formats are taken into account. Since first order edge mask is the oldest technique only second and third order masks are considered. As a first step original sample image is degraded with random noise.

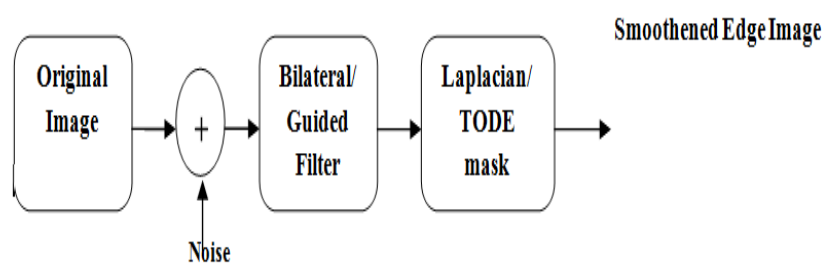

Fig.7.Block diagram of proposed work

Secondly noisy image is smoothened using bilateral and guided filter. Finally edge map is derived using second order edge mask Laplacian and third order edge mask TODE. Parameters such as Peak Signal to Noise Ratio (PSNR), Mean Square Error (MSE) and Entropy were analyzed. Fig.7 shows the operation of the newly proposed work. Experimental results show that the guided filter smoothing is best suited edge preserving smoothing filter than the bilateral filter.

\section{EXPERIMENTAL RESULTS}

To analyze the result and compute the performance metric, the proposed technique is implemented using MATLAB software. Natural fruits.gif and cat.bmp, remote sensing street.jpg images are subjected for testing with newly proposed approach. Result showing that the guided filter is the best suitable filter for edge preserving smoothing than bilateral filter. Also Third Order Edge mask (TODE) and guided smoothing technique produces better edge map for further processing.

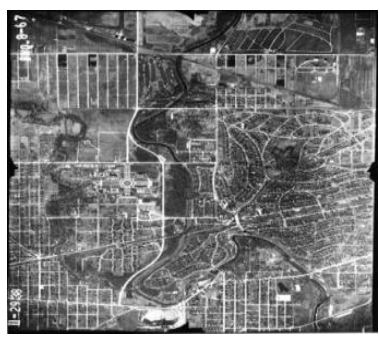

Fig.8a.Original Image

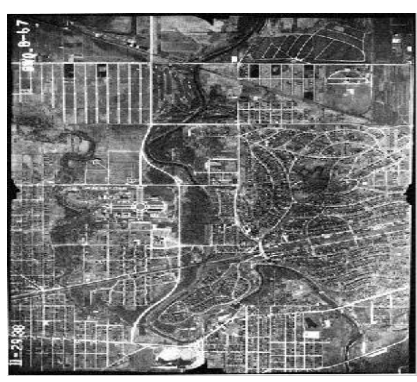

Fig.8b.Noisy Image 


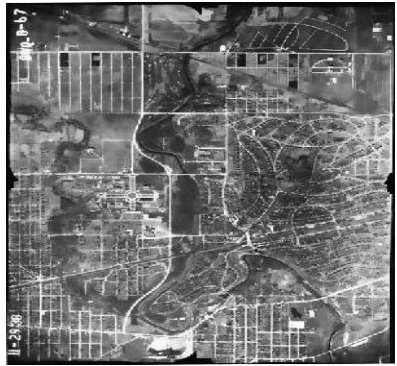

Fig.8c. Bilateral Smoothened Image

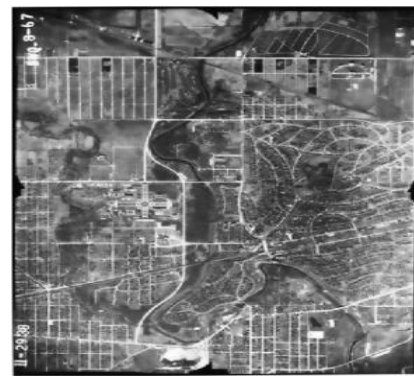

Fig.8d. Guided Smoothened Image

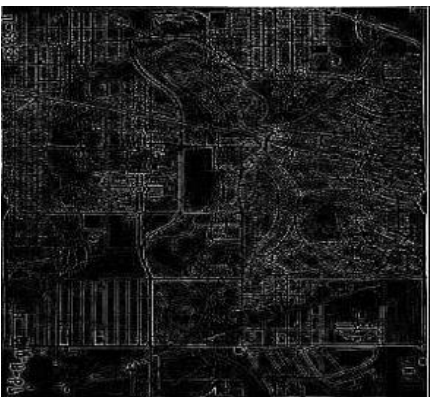

Fig.8e. Bilateral filtered Laplacian

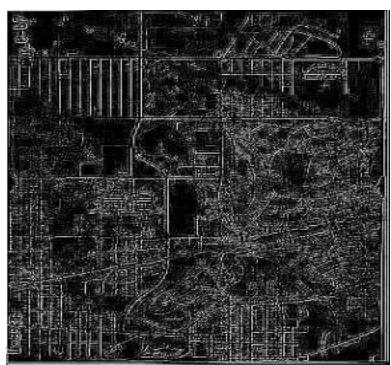

Fig.8f. Bilateral filtered TODE Image

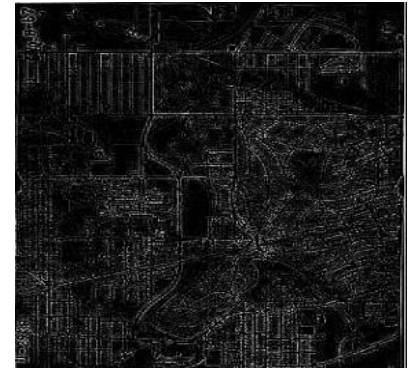

Fig.8g. Guided filtered Laplacian Image

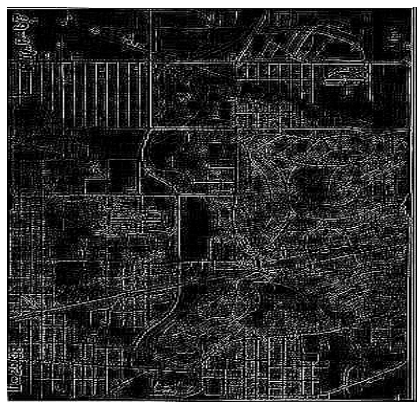

Fig.8h. Guided filtered TODE Image

Above images depicted in fig.8a through fig. $8 \mathrm{~h}$ shows the result of proposed technique for the remote sensing street.jpg image, that includes original image, noisy image, Bilateral smoothened image, Guided smoothened image, Bilateral filtered Laplacian image, Bilateral filtered TODE image ,Guided filtered Laplacian image and Guided filtered TODE image.

Performance comparisons of bilateral and guided filter for second order Laplacian edge mask for different images given in Table1. Metrics such as PSNR, MSE and Entropy were measured. Higher PSNR differentiate high quality image. Since PSNR and MSE are inversely proportional, MSE should be as low as possible. Entropy is a statistical measure of randomness used to describe texture of the image. Result proves that guided filter produces higher PSNR, Lower MSE and smaller Entropy for all types of image samples and file formats taken for analysis. For an example remote sensing street image the guided filter offers Peak Signal to Noise Ratio of 7.1615 which is higher than that of bilateral filter output 7.0865.Mean Square Error of $1.2500 \times 10^{4}$ is obtained from guided filter, whereas $1.2718 \times 10^{4}$ in case of bilateral filter. Likewise the guided filter has higher entropy than bilateral filter. These statements are true for other sample images too.

Table. 1 Second order edge mask (Laplacian)

\begin{tabular}{|c|c|c|c|c|c|c|}
\hline \multirow{2}{*}{$\begin{array}{c}\text { Image } \\
\text { types }\end{array}$} & \multicolumn{3}{|c|}{ Bilateral filter } & \multicolumn{3}{c|}{ Guided Filter } \\
\cline { 2 - 7 } & PSNR & MSE & ENTROPY & PSNR & MSE & ENTROPY \\
\hline Fruits.gif & 7.9595 & $1.0402 \times 10^{4}$ & 1.6366 & 7.9871 & $1.0336 \times 10^{4}$ & 1.2491 \\
\hline Street.jpg & 7.0865 & $1.2718 \times 10^{4}$ & 2.8312 & 7.1615 & $1.2500 \times 10^{4}$ & 2.5611 \\
\hline Cat.bmp & 7.2880 & $1.2142 \times 10^{4}$ & 1.8951 & 7.3283 & $1.2030 \times 10^{4}$ & 1.3482 \\
\hline
\end{tabular}


Similarly Table.2 shows the comparison of bilateral and guided filter for third order edge mask (TODE). Results prove that guided filter is a best edge preserving smoothing filter for imaging field subjected to PSNR, MSE and Entropy. For an example, fruits.gif image results provide PSNR of 8.1351 in case of guided filter smoothing which is higher than that of bilateral smoothing and MSE of guided filter smoothing is $9.9901 \times 10^{3}$ which is smaller than that of bilateral smoothing whose value is $1.0037 \times 10^{4}$. Also for other sample images the guided filter offers higher PSNR and Lower MSE values.

Table. 2 Third Order Edge Mask (TODE)

\begin{tabular}{|c|c|c|c|c|c|c|}
\hline \multirow{2}{*}{$\begin{array}{l}\text { Image } \\
\text { types }\end{array}$} & \multicolumn{3}{|c|}{ Bilateral filter } & \multicolumn{3}{|c|}{ Guided Filter } \\
\hline & $P S N R$ & $M S E$ & ENTROPY & $P S N R$ & $M S E$ & ENTKOPY \\
\hline Fruits.gif & 8.1146 & $1.0037 \times 10^{4}$ & 1.9525 & 8.1351 & $9.9901 \times 10^{3}$ & 1.6168 \\
\hline Street.jpg & 7.4172 & $1.1786 \times 10^{4}$ & 2.8479 & 7.4334 & $1.1742 \times 10^{4}$ & 2.8644 \\
\hline Cat.bmp & 7.4778 & $1.1622 \times 10^{4}$ & 2.1065 & 7.4936 & $1.12119 \times 10^{4}$ & 1.6030 \\
\hline
\end{tabular}

By comparing table 1 and table 2 details, it is well understood that the combination of guided filter smoothing and third order edge mask TODE performs better results in retrieving the edges while smoothing. Thus the guided filter is best suited for third order edge mask.

Graphical representation of the performance analysis depicted in following figures. Fig .9 shows PSNR performance and fig.10 represents MSE performance of second order edge mask using bilateral and guided filter. Similarly fig.11and fig. 12 shows the results of third order edge mask (TODE) for PSNR and MSE respectively.

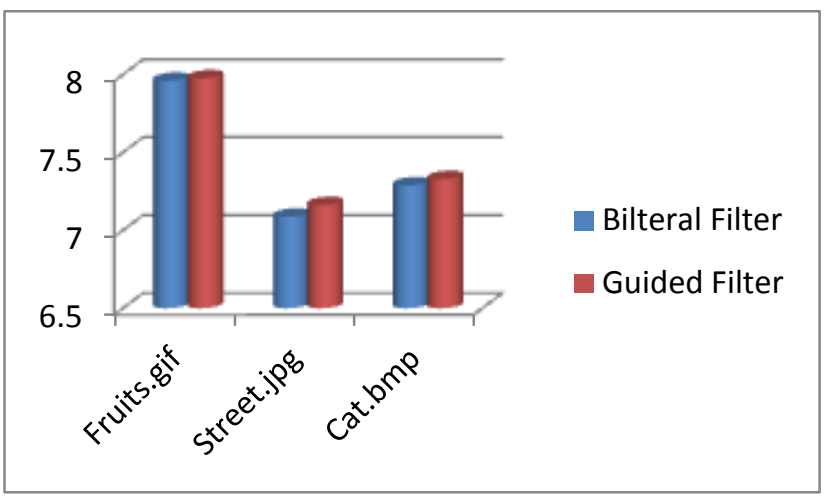

Fig.9.Peak Signal to Noise Ratio - Second order edge mask (Laplacian)

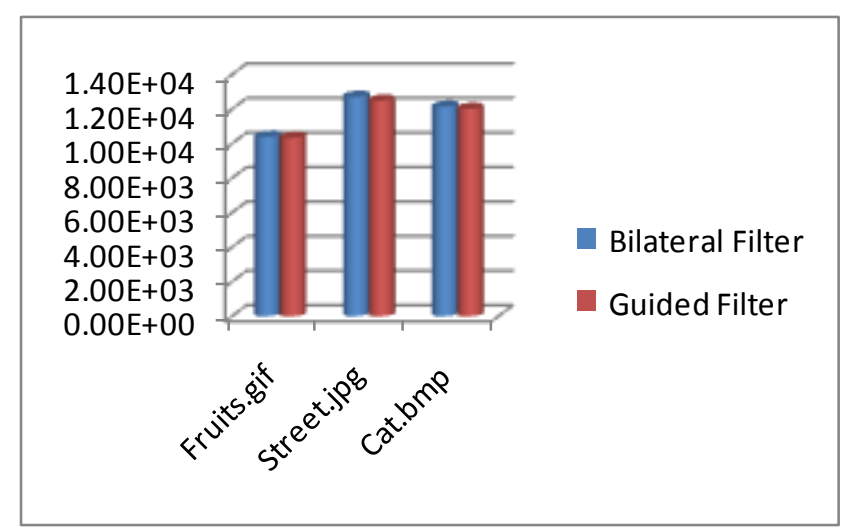

Fig.10.Mean Square Error-Second order edge mask (Laplacian)

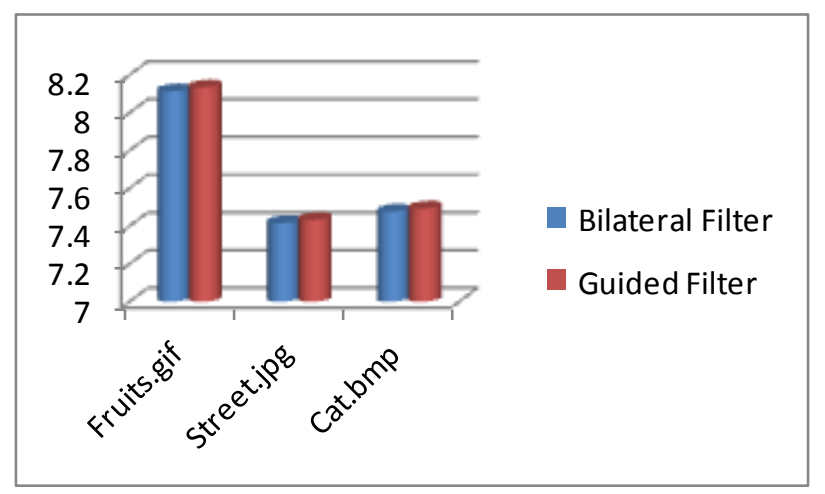

Fig.11.Peak Signal to Noise Ratio -Third order edge mask (TODE)

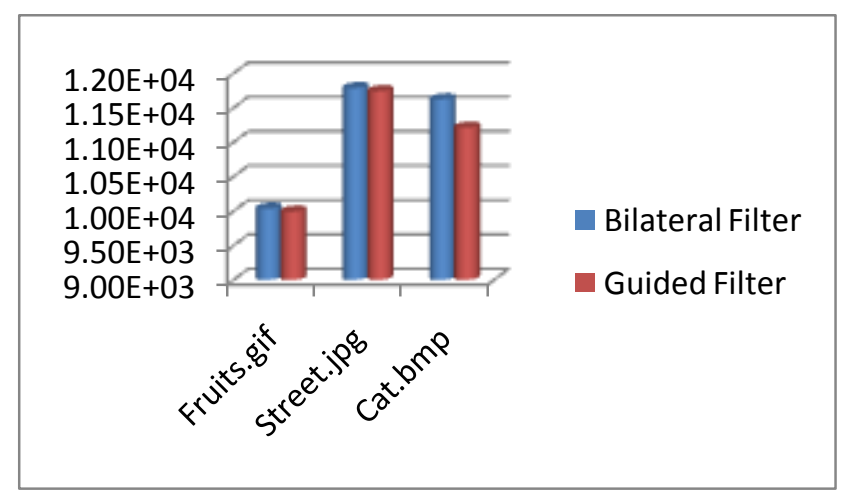

Fig.12. Mean Square Error -Third order edge mask (TODE)

\section{CONCLUSION AND FUTURE WORK}

Image smoothing and edge detections are the prominent image processing steps for machine vision applications. Noises are random in nature.It disturb the images during sensing and transmission. This paper attempts to find out the better edge preserving smoothing techniques for third order edge mask.First order edge mask is simple to implement but contains two different masks that increases computational time and is a too old process to extract edges.Second order Laplacian contains single mask producing thin edges and hence good localization towards edges.To improve the performance of the edge detection further a third order mask is used. Especially in noisy environments the third order mask enhances both edges and noise.Therefore a suitable smoothing process is required prior to the application of edge mask.In this paper state of art the methods of edge preserving smoothing techniques like non linear bilateral filter and linear guided filter operated on variety of images and storage formats in both second order and third order edge mask.Results showing that the guided filter smoothing is best suitable technique with higher degree of edge preserving quality. Guided filter based third order mask offers very high PSNR, very minima MSE and higher entropy.In future this proposed guided filter based third order edge mask is applied to extract the edge features for registration and image segmnetation processes.In addition this approach will be tested on medical images for accurate edge measurements. Parameters such as Structural Similarity index (SSIM), Pratt's figure of merit(PFOM) are measured to improve the performance of the proposed technique. Also this proposed approach is extended to multi spectral and video images directy to extract edge information. 


\section{REFERENCES}

[1] Rafael C. Gonzalez and Richard E. Woods,2001 Digital Image Processing.

[2] Mr.Avadhoot R. Telepatil, S.A.Patil,Mr.Vishal P.Paramane, A Survey on Median Filters for Removal of High Density Salt \& Pepper Noise in Noisy Image, IOSR-JECE. pp 22-26.

[3] Ardeshir Goshtasby and Martin Satter. An Adaptive Window Mechanism for Image Smoothing.

[4] Chapter3, FILTERS, www.bioss.ac.uk/people/chris

[5] B.Thamotharan,

M.Menaka,

Sandhya Vaidyanathan,Sowmya Ravikumar, 2012, Survey on image processing in the field of de-noising techniques and edge detection techniques on radiographic images, Journal of Theoretical and Applied Information Technology, Vol. 41 No.1,pp-26-34.

[6] Govindaraj.V, Sengottaiyan.G,2013, Survey of Image Denoising using Different Filters, IJSETR,Volume 2, Issue 2, ,pp.344-351.

[7] Amita Kumari. Pankaj Dev Chadha, 2014 A Survey on Filtering Technique for Denoising Images in Digital Image Processing, International Journal of Advanced Research in Computer Science and Software Engineering, Volume 4, Issue 8, pp-612-614.

[8] T. Joel and R. Sivakumar, Despeckling of Ultrasound Medical Images: 2013 A Survey, Journal of Image and Graphics Vol. 1, No. 3, pp-161-165

[9] Vinh Hong, Henryk Palus, Dietrich Paulus, Edge Preserving Filters on Color Images,

[10] Faten A. Dawood, Rahmita W. Rahmat, Suhaini B. Kadiman, Lili N. Abdullah, Mohd D. Zamrin,2012, World Academy of Science, Engineering and Technology Vol:6, International Scholarly and Scientific Research \& Innovation 6(9) pp-415-420

[11] Giuseppe Papari, Nicolai Petkov, and Patrizio Campisi, Artistic Edge and Corner Enhancing Smoothing,2007, IEEE Trans. on image processing, VOL. 16, NO. 10,pp2449-2462.

[12] Michael Elad, 2002, On the origin of bilateral filter and the ways to improve it, IEEE trans. on image processing,Vol.11,NO.10,pp-1141-1151.

[13] Kaiming He, Jian Sun, Xiaoou Tang,2013, IEEE trans. on pattern analysis and machine intelligence, vol. 35, no. 6, pp-1397-1409

[14] Tianshuang Qiu, Aiqi Wang, Nannan Yu, and Aimin Song,2013, LLSURE: Local Linear SURE-Based EdgePreserving Image Filtering, IEEE trans. on image processing, vol. 22, NO. 1.
[15] Gurjeet Kaur,Merge $\mathrm{L}_{0}$ minimization technique, 2015, International Journal of Advanced Research in Computer Science and Software Engineering,Vol.5,Issue.2,pp-857861.

[16] Charles Boncelet,2005, "Image Noise Models" in Alan C.Bovik, Handbook of Image and Video Processing, ,pp397-409

[17] Chandrika Saxena, Prof. Deepak Kourav, 2014,Noises and Image Denoising Techniques: A Brief Survey, International Journal of Emerging Technology and Advanced Engineering, Volume 4, Issue 3,pp-878-885.

[18] Mohd Awais Farooque, Jayant S.Rohankar, 2013, Survey on various noises and techniques for denoising the color image, IJAIEM, Volume 2, Issue 11,pp-217-221.

[19] Rupinderpal Kaur, Rajneet Kaur,2013, Survey of Denoising Methods Using Filters and Fast Wavelet Transform, International Journal of Advanced Research in Computer Science and Software Engineering, Volume 3, Issue 2, pp-133-136.

[20] Pawan Patidar, Manoj Gupta, Sumit Srivastava, Ashok Kumar Nagawat,2010, Image De-noising by Various Filters for Different Noise,IJCA, Volume 9- No.4,,pp45-50

[21] James Church, Dr. Yixin Chen, and Dr. Stephen Rice, 2008,A Spatial Median Filter for Noise Removal in Digital Images, IEEE, pp. 618-623.

[22] Toran Lal Sahu, Mrs. Deepty Dubey, 2012,A Survey on Image Noises and Denoise Techniques, IJARCET, Volume 1, Issue 9, pp.77-81.

[23] Kaiming He,Jian Sun,Xiaoou Tang, 2013,Guided Image Filtering, IEEE transaction on pattern analysis and machine intelligence,Vol.35,No.6,pp.1397-1409.

[24] Chandrajit Pal,Amlan Chakrabarti,Ranjan Ghosh, 2015,A brief survey on recent edge preserving smoothing algoriths on digital images.Elsevier ,Procedia Computer Science.

[25] G.J.Joyce Mary,A.Rijuvana Begum, 2015, Edge Detection using Third Order Difference Equation: A New Dimension, Communications on Applied Electronics,vol.1.No.6,pp.10-14.

[26] S.Asha, R.Rajesh Kanna, 2014,A Survey on Content Based Image Retrieval Based On Edge Detection, IJCSIT, Vol. 5 (6) , pp.8272-8275.

[27] Akansha Mehrotra, Krishna Kant Singh, M.J.Nigam, 2012, A Novel Algorithm for Impulse Noise Removal and Edge Detection, International Journal of Computer Applications (0975 - 8887) Volume 38- No.7,pp-30-34. 